\title{
ADAMTS14 gene polymorphism associated with knee osteoarthritis in Thai women
}

\author{
T. Poonpet ${ }^{1,2}$, S. Honsawek ${ }^{3}$, N. Tammachote ${ }^{4}$, S. Kanitnate ${ }^{4}$, \\ R. Tammachote ${ }^{2}$
}

${ }^{1}$ Biological Science Program, Faculty of Science, Chulalongkorn University, Bangkok, Thailand

${ }^{2}$ Department of Botany, Faculty of Science, Chulalongkorn University, Bangkok, Thailand

${ }^{3}$ Department of Biochemistry, Faculty of Medicine, Chulalongkorn University, Bangkok, Thailand

${ }^{4}$ Department of Orthopedic Surgery, Faculty of Medicine, Thammasat University, Pathumthani, Thailand

Corresponding author: R. Tammachote

E-mail: Ratchaneekorn.T@chula.ac.th

Genet. Mol. Res. 12 (4): 5301-5309 (2013)

Received December 12, 2012

Accepted April 4, 2013

Published November 7, 2013

DOI http://dx.doi.org/10.4238/2013.November.7.5

\begin{abstract}
Osteoarthritis (OA) is the most prevalent form of arthritis in the elderly. This disease is characterized by breakdown and loss of articular cartilage due to genetic, mechanical and environmental factors. Although the pathophysiology of OA is not completely known, several candidate genes have been reported to be associated with OA susceptibility. We assessed the association between genetic variation in the ADAMTS14 region and knee osteoarthritis susceptibility in the Thai population. The rs4747096 SNP was genotyped by PCR-RFLP on genomic DNA extracted from peripheral blood of $108 \mathrm{OA}$ patients and 119 controls. The PCR product (196 bp) was digested with BspEI. A sample with the GG genotype showed two band sizes of 158 and $38 \mathrm{bp}$, while a sample with the AA genotype showed a single band size of $196 \mathrm{bp}$. Heterozygotes with the AG genotype showed all three corresponding bands. Genotype distributions,
\end{abstract}


allele frequencies and model of inheritance in patients and controls were compared. In females, the frequency of the AA genotype and the A allele were significantly higher in knee OA patients than in controls [odds ratio $(\mathrm{OR})=2.79,95 \%$ confidence interval $(\mathrm{CI})=1.05-7.59$ and $\mathrm{OR}=1.58$, $95 \% \mathrm{CI}=1.00-2.45$, respectively]. Moreover, genotypic AA and AG were associated with significantly increased risk for knee OA when compared to $\mathrm{GG}(\mathrm{OR}=2.72,95 \% \mathrm{CI}=1.10-6.87)$. No significant associations were observed in males. In conclusion, the nsSNP rs4747096 in ADAMTS14 was associated with knee $\mathrm{OA}$ in female Thai patients; therefore, the role of ADAMTS14 in OA seems to be gender-dependent.

Key words: Osteoarthritis; ADAMTS14; rs4747096; PCR-RFLP; Thai population

\section{INTRODUCTION}

Osteoarthritis (OA), a degenerative joint disorder, is the most prevalent form of arthritis in the elderly, which causes pain and restricted motion in joints, particularly the knee and hip (Dieppe and Lohmander, 2005). This disease is characterized by the breakdown and loss of the articular cartilage in parallel with subchondral bone sclerosis, joint space narrowing, and bony outgrowths at the joint margins (osteophytes) (Dieppe and Kirwan, 1994). The multifactorial nature of OA is well accepted, with several attributing factors including a variety of non-genetic factors (obesity, female gender, advanced age, and joint injury) and a combinatorial effect of weak genetic factors (Spector and MacGregor, 2004; Teichtahl et al., 2005; Ikegawa, 2007). Although the pathogenesis of this complex disease is not entirely known, various candidate genes and linkage regions have been shown to be associated with OA susceptibility (Valdes and Spector, 2009). A number of genes involved in developmental processes or maintenance of cartilage and bone have been found to be associated with OA occurrence and progression (Tammachote, 2011). OA-associated genes were categorized into five molecular pathways including inflammation, extracellular matrix (ECM) molecules, Wnt signaling, osteocyte modulation or chondrocyte differentiation, and proteases and their inhibitors (Valdes and Spector, 2009).

Osteoarthritic joints have an imbalance between synthesis and degradation of ECM leading to cartilage destruction. Therefore, metalloproteinases have been considered as the major groups of enzymes responsible for this OA hallmark (Kevorkian et al., 2004; Murphy and Nagase, 2008). A disintegrin and metalloproteinases with thrombospondin motifs (ADAMTSs), members of the $\mathrm{Zn}^{2+}$-metalloproteinase superfamily (metzincins), are of interest as novel genetic factors of OA (Murphy and Nagase, 2008). The proteinases in the ADAMTS family resemble and share a common domain structure with the proteinases in the ADAM (A disintegrin and metalloproteinase) family in having a prodomain, an adamalysin/reprolysinlike catalytic domain, a disintegrin-like domain and a cysteine-rich domain. However, the ADAMTS proteinases differ from ADAM proteinases in lacking transmembrane domains and are a putative secretory protein. In addition, ADAMTSs possess a thrombospondin type 1 (TSP1) motif, followed by a spacer region and a variable number of TSP1 repeats, which appear to be involved in binding to ECM components, such as aggrecan and procollagen (Kuno et al., 1997; Hurskainen et al., 1999; Apte, 2004). ADAMTS14, which is located on 
chromosome 10q22.1, has been recently reported to be a novel OA candidate gene (RodriguezLopez et al., 2009). A non-synonymous single nucleotide polymorphism (nsSNP) of likely deleterious effect in exon 21 of ADAMTS14 (rs4747096) was demonstrated to be associated with knee OA. Rodriguez-Lopez et al. found that the $\mathrm{G}$ allele in rs4747096 was associated with women requiring total knee replacement because of OA and with symptomatic hand OA in the Caucasian population (Rodriguez-Lopez et al., 2009).

To our knowledge, there has been no further published report about the role of this SNP in other populations. Since several studies have indicated the site-specific, sex-specific and ethnic-specific nature of genetic effects on osteoarthritis, this research aimed to analyze the association of rs4747096 nsSNP in ADAMTS14 with knee OA susceptibility in a Thai population in a case-control study manner.

\section{MATERIAL AND METHODS}

\section{Study population}

This study has been approved by the Institutional Review Board of the Faculty of Medicine, Thammasat University. Written informed consent was received from all subjects prior to their participation in the study. The 108 cases ( 88 women and 20 men; mean age 70 years, range 51-91 years) were patients with severe primary knee OA undergoing total knee replacement (TKR) surgery. The indications of TKR were the absence of femoral joint space, no responses to conservative treatment longer than six months, and grades 3-4 of radiographic signs of OA according to the Kellgren-Lawrence grading system (Kellgren and Lawrence, 1957). The control population consisted of 119 healthy individuals (93 women and 26 men; mean age 55 years, range 50-60 years). All were diagnosed by physicians to have no symptoms or signs of OA, other arthritis or any joint disorders (chronic pain, inflammation, tenderness, swelling or limitation of movement), and to have no family history of those diseases. The patients with any systemic inflammatory or autoimmune disorders, or any type of malignant or chronic illness were not included in this study.

\section{Sample collection and DNA extraction}

Peripheral blood samples were taken from each participant by standard venipuncture. All blood samples were kept in ethylenediaminetetraacetic acid (EDTA)-containing tubes at $4^{\circ} \mathrm{C}$ until use. Genomic DNA was extracted from whole blood with innuPREP Blood DNA Master kit (Analytikjena, Seerasen, Eisfeld, Germany). The DNA concentration was estimated by measuring the absorbance at $260 \mathrm{~nm}$. Afterwards, DNA was diluted to $50 \mathrm{ng} / \mu \mathrm{L}$ and used as the template in SNP genotyping.

\section{SNP genotyping}

The rs4747096 polymorphism was genotyped by the polymerase chain reactionrestriction fragment length polymorphism (PCR-RFLP). The mutated forward primer (5'-GGAACTTGGGACGCCAGAGGGGCAGTGGGTGCCACAATCCG-3') and the reverse primer (5'-AGGGAGGTGAAGGTCACACA-3') were used for amplification of the area of interest. PCR was conducted in a $20-\mu \mathrm{L}$ volume containing $2 \mu \mathrm{L} 10 \mathrm{X}$ Taq buffer, $1.5 \mathrm{mmol}$ 
$\mathrm{MgCl}_{2}, 0.5$ mmol dNTPs, $0.2 \mu \mathrm{mol}$ of each primer, $100 \mathrm{ng}$ genomic DNA template, and $0.4 \mathrm{U}$ Taq DNA polymerase (Fermentas Inc., Hanover, NH, USA). The PCR program consisted of the following steps: initial denaturation of $5 \mathrm{~min}$ at $94^{\circ} \mathrm{C}$, followed by 35 cycles of denaturation at $94^{\circ} \mathrm{C}$ for $30 \mathrm{~s}$, annealing at $54^{\circ} \mathrm{C}$ for $30 \mathrm{~s}$, and extension at $72^{\circ} \mathrm{C}$ for $1 \mathrm{~min}$, and a final extension of $72^{\circ} \mathrm{C}$ for $5 \mathrm{~min}$. The PCR product (196 bp) was incubated overnight at $37^{\circ} \mathrm{C}$ with the $B s p \mathrm{EI}$ restriction enzyme (New England BioLabs, Beverly, MA, USA), which can cut PCR products with the $\mathrm{G}$ allele. After incubation, the mixtures were electrophoresed on $3 \%$ agarose and visualized by ethidium bromide staining and ultraviolet light transillumination. The expected fragment was 196 bp in AA, 158 and 38 bp in GG and 196, 158 and 38 bp in AG genotypes, respectively. For accuracy testing, several samples with different genotypes were sequenced.

\section{Statistical analysis}

Comparison of demographic data was conducted by Excel 2007 (Microsoft ${ }^{\circledR}$ Excel $\left.^{\circledR}\right)$. The Chi-square test was applied for categorical data, and the unpaired Student $t$-test was applied for continuous data. Genotype and allele proportions in patient and control groups were evaluated for Hardy-Weinberg equilibrium (HWE) as described at the following website: http://www.oege.org/ software/hardy-weinberg.html (Rodriguez et al., 2009). Genotype distributions, allele frequencies in OA patients and controls, and model of inheritance were determined by the StatCalc program (AcaStat Software, Leesburg, VA, USA). $\mathrm{P}<0.05$ was considered to be statistically significant. The risk associated with individuals' genotypes and alleles was evaluated as the odds ratio (OR) with $95 \%$ confidence intervals $(95 \% \mathrm{CI})$. For gender stratification analysis, female OA patients were compared with female controls and male OA patients with male controls.

\section{RESULTS}

Demographic characteristics and the number of individuals in each group of the population studied are illustrated in Table 1. In this study, there were no significant differences between OA patients and healthy controls in terms of gender, weight, height, and body mass index (BMI). However, the average age of the knee OA group was significantly greater than that of controls.

\begin{tabular}{lccc}
\multicolumn{4}{c}{ Table 1. Characteristics of osteoarthritis (OA) patients and control individuals. } \\
\hline Variables & Knee OA patients (N = 108) & Controls (N = 119) & P \\
\hline Female (\%) & $88(81.5)$ & $93(78.2)$ & 0.53 \\
Age [years mean (SD)] & $69.94(8.41)$ & $54.50(2.72)$ & $<0.001^{*}$ \\
Weight [kg mean (SD)] & $63.57(10.92)$ & $63.48(12.11)$ & 0.95 \\
Height [cm mean (SD)] & $155.59(7.05)$ & $157.18(6.25)$ & 0.06 \\
BMI [kg/m² mean (SD)] & $26.29(4.45)$ & $25.65(4.25)$ & 0.27 \\
\hline
\end{tabular}

*Statistically significant.

The rs4747096 polymorphism was genotyped by PCR-RFLP. PCR product size was 196 bp. BspEI cleaved the PCR product with G allele into 158- and 38-bp fragments. An example of an electrophoretic gel displaying PCR products after digestion with BspEI is shown in Figure 1. As the smallest fragment ( $38 \mathrm{bp}$ ) was difficult to be visualized, the presence of a 196-bp fragment corresponded to the AA genotype, the 158-bp fragment corresponded to the GG genotype, and the 196- and 158-bp fragments corresponded to the AG genotype. 


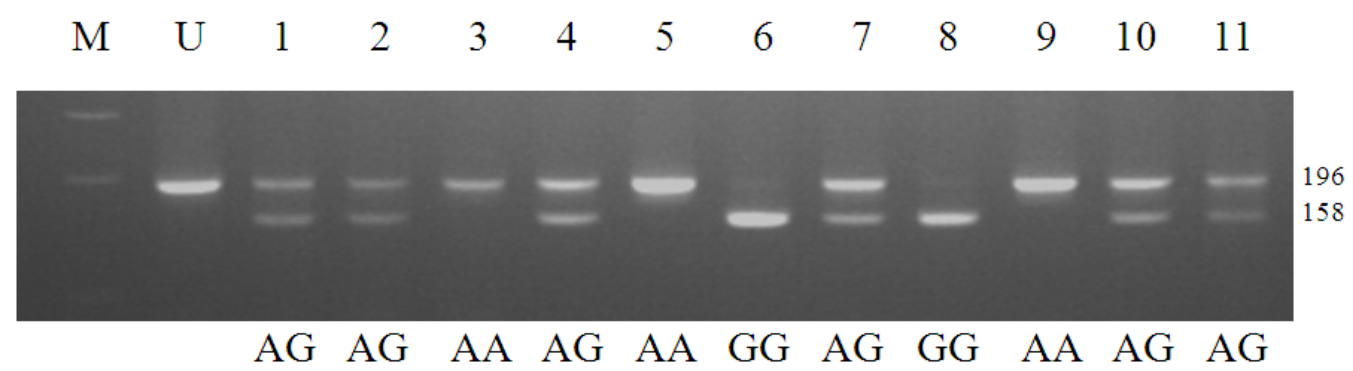

Figure 1. ADAMTS14 rs4747096 genotyping by PCR-RFLP. The PCR product was digested with BspEI, which cleaved the PCR products with G alleles, and subjected to electrophoresis on a 3\% agarose gel. Lane $M=100-\mathrm{bp}$ DNA standard marker; lane $U=$ uncut PCR product; lanes 1 to $11=$ sample 1-11. Genotypes of each sample are shown in the bottom.

The genotype and allelic frequencies of rs4747096 are summarized in Table 2. Interestingly, the genotype distribution among the controls was not in Hardy-Weinberg equilibrium (HWE) due to the overrepresentation of the rare homozygous genotype (GG), whereas the genotypes of patients were found to be in HWE ( $\mathrm{P}>0.05)$. AA was the most common genotype in OA patients and normal controls ( 46.3 and $40.3 \%$, respectively). We found a significant association between the AA genotype and knee OA in female patients $(\mathrm{P}=0.023)$. This genotype was linked to a higher risk of knee $\mathrm{OA}$ in females $(\mathrm{OR}=2.79,95 \% \mathrm{CI}=1.05-7.59)$. In addition, the A allele was also significantly associated with female knee $\mathrm{OA}$ patients $(\mathrm{OR}=1.58 ; 95 \% \mathrm{CI}=1.00-2.45$; $\mathrm{P}=0.038$ ). Moreover, a nearly significant association was found between the AG genotype and knee $\mathrm{OA}$ in female patients $(\mathrm{OR}=2.65 ; 95 \% \mathrm{CI}=0.99-7.19 ; \mathrm{P}=0.031)$. In contrast, the rs4747096 polymorphism was not associated with OA susceptibility in males in this study.

Table 2. Genotype distribution and allelic frequency of ADAMTS14 polymorphism between knee osteoarthritis (OA) patients and normal controls.

\begin{tabular}{|c|c|c|c|c|}
\hline rs4747096 & $\mathrm{OA}$ & Control & OR $(95 \% \mathrm{CI})$ & $P$ \\
\hline \multicolumn{5}{|l|}{ Genotype (\%) } \\
\hline \multicolumn{5}{|l|}{$\mathrm{AA}$} \\
\hline Whole group & $50(46.3 \%)$ & $48(40.3 \%)$ & $2.17(0.92-5.19)$ & 0.054 \\
\hline Female & $40(45.5 \%)$ & $35(37.6 \%)$ & $2.79(1.05-7.59)$ & 0.023 \\
\hline Male & $10(50.0 \%)$ & $13(50.0 \%)$ & $0.77(0.09-6.35)$ & 0.56 \\
\hline \multicolumn{5}{|l|}{$\mathrm{AG}$} \\
\hline Whole group & $46(42.6 \%)$ & $46(38.7 \%)$ & $2.08(0.87-5.02)$ & 0.07 \\
\hline Female & $39(44.3 \%)$ & $36(38.7 \%)$ & $2.65(0.99-7.19)$ & 0.031 \\
\hline Male & $7(35.0 \%)$ & $10(38.5 \%)$ & $0.70(0.07-6.39)$ & 0.54 \\
\hline \multicolumn{5}{|l|}{ GG } \\
\hline Whole group & $12(11.1 \%)$ & $25(21.0 \%)$ & $1 *$ & \\
\hline Female & $9(10.2 \%)$ & $22(23.7 \%)$ & $1 *$ & \\
\hline Male & $3(15.0 \%)$ & $3(11.5 \%)$ & $1 *$ & \\
\hline \multicolumn{5}{|l|}{ Allele (\%) } \\
\hline \multicolumn{5}{|l|}{ A } \\
\hline Whole group & $146(67.6 \%)$ & $142(59.7 \%)$ & $1.41(0.94-2.11)$ & 0.08 \\
\hline Female & $119(67.6 \%)$ & $106(57.0 \%)$ & $1.58(1.00-2.45)$ & 0.038 \\
\hline Male & $27(67.5 \%)$ & $36(69.2 \%)$ & $0.92(0.35-2.46)$ & 0.86 \\
\hline \multicolumn{5}{|l|}{$\mathrm{G}$} \\
\hline Whole group & $70(32.4 \%)$ & $96(40.3 \%)$ & $1 *$ & \\
\hline Female & $57(32.4 \%)$ & $80(43.0 \%)$ & $1 *$ & \\
\hline Male & $13(32.5 \%)$ & $16(30.8 \%)$ & $1 *$ & \\
\hline
\end{tabular}

*Reference. 
As the SNP showed significant association only with females, the model of inheritance was not analyzed in the whole group and men group. The result from females showed that this polymorphism is inherited by an autosomal dominance aspect, since the AA and AG genotypes significantly increased risk of the disease $(\mathrm{OR}=2.72,95 \% \mathrm{CI}=1.10-6.87, \mathrm{P}=$ 0.017), whereas the AA genotype showed no significant difference (Table 3 ).

\begin{tabular}{|c|c|c|c|c|}
\hline \multirow[t]{2}{*}{ Model of inheritance } & \multicolumn{4}{|c|}{ Female } \\
\hline & OA & Control & OR $(95 \% \mathrm{CI})$ & $P$ \\
\hline \multicolumn{5}{|c|}{ A dominance, $\mathrm{G}$ wild type } \\
\hline $\mathrm{AA}$ or $\mathrm{AG}$ & $79(89.8 \%)$ & $71(76.3 \%)$ & $2.72(1.10-6.87)$ & 0.017 \\
\hline GG & $9(10.2 \%)$ & $22(23.7 \%)$ & $1 *$ & \\
\hline \multicolumn{5}{|l|}{ A recessive, $G$ wild type } \\
\hline AA & $40(45.5 \%)$ & $35(37.6 \%)$ & $1.38(0.73-2.61)$ & 0.29 \\
\hline $\mathrm{GG}$ or $\mathrm{AG}$ & $48(54.5 \%)$ & $58(62.4 \%)$ & $1 *$ & \\
\hline
\end{tabular}

*Reference.

\section{DISCUSSION}

ADAMTS is a family of zinc-dependent proteases that are implicated in a variety of normal and pathological conditions, including arthritis. ADAMTS14 belongs to the ADAMTS family. There are 19 published vertebrate family members of ADAMTSs, numbered 1-10 and 12-20 to date (Cal et al., 2002; Somerville et al., 2003). Although little is still known about its function, ADAMTS14 has high similarities in sequence and domain structure to ADAMTS-2 (56\%) and ADAMTS-3 (63\%) (Colige et al., 2002), which play a major role in the biosynthesis of collagen precursors. There is evidence that these three enzymes have the ability to cleave the N-propeptides of procollagen type I (pNPI activity) and the homotrimeric procollagen type II before they are incorporated into collagen fibers (Lapiere et al., 1971; Tuderman et al., 1978; Hojima et al., 1989, 1994) . Moreover, ADAMTS14 has been shown to have pNPI activity in vitro, and has been suggested as a possible source of residual pNPI activity observable in the bone, tendon, cartilage, skin, and other tissues of Ehlers-Danlos syndrome type VIIC patients, dermatosparaxic cattle, and Adamts2-null mice (Fernandes et al., 2001; Colige et al., 2002; Le Goff et al., 2006).

In the present study, we evaluated the association between rs4747096 nsSNP in ADAMTS14, a recently discovered OA candidate gene, and presence of knee OA in a Thai

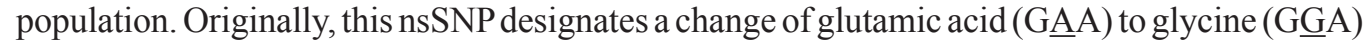
in the $\mathrm{COOH}$-terminal domain of ADAMTS14 protein. The allele $\mathrm{G}$ is the ancestral allele, as it exists in ADAMTS14 orthologues in nonhuman primates such as chimpanzee, whereas the A allele is more common in humans than the G allele (Sherry et al., 2001). Recently, there was evidence of the T allele, which determines the amino acid valine (GIA) in the rs 4747096 database. The existence of the new allele seems to complicate the analysis of rs 4747096 role in OA. However, this new variation was discovered in genomic DNA from cancer patients (Futreal et al., 2004), and was not apparent in any population in The 1000 Genome Project (2010), including the Asian population even though the whole genome sequencing method was used as genotyping technique. Thus, the T allele was neglected in this study. 
Genotype distributions and allele frequencies in a Thai population showed an opposite trend compared to a previous study (Rodriguez-Lopez et al., 2009). The result from this study demonstrated that rs4747096 nsSNP was associated with susceptibility to knee OA in female patients undergoing total knee replacement surgery. The AA genotype carried the highest risk in comparison with the other genotypes. Moreover, the analysis for model of inheritance indicated that the A allele increased the risk of knee OA in women. In contrast, Rodriguez-Lopez et al. study (2009) reported that the G allele was overrepresented and associated with knee OA in Caucasian women. The reason for these contradictory results may be due to differences in ethnicity or shared environmental factors. However, the non-associated results from male population are consistent. Several studies have also revealed contradictory findings of genetic contribution to OA. For example, a polymorphism in $I L 1 R N$ was reported to be associated with disease severity in knee OA in a Caucasian population (Attur et al., 2010), while a study in a Turkish population found no association (Sezgin et al., 2007).

It was believed that the substitution of glutamic acid (acidic) to glycine (nonpolar) in ADAMTS14 (E1049G) may result in abnormal ability of the protein in processing collagen precursor and finally leading to OA. The positive results of both $\mathrm{A}$ and $\mathrm{G}$ alleles obtained from the present and previous studies may indicate that the amino acid substitution determined by rs4747096 in ADAMTS14 is not the direct damaging factor contributing to OA. However, this polymorphic locus may be linked to the OA causative gene on the same chromosome and the SNP is inherited jointly with the disease.

The number of the ancestral G-allele in Thais was higher than in Caucasians, especially in women (38vs 20\%). These figures are consistent with HapMap reports showing that the $\mathrm{G}$ allele frequency of this SNP in the East Asian population and European population was approximately 37 and 19\%, respectively (The International HapMap Consortium, 2003). The genotype distribution of the control group in this study did not fit HWE, which indicated that specific disturbing influences were introduced. One possible influence is a selective pressure for the G allele, since the GG genotype seemed to be the protective genotype. As Thais are a mixed racial population, gene flow or migration can also be influences that alter the existing gene frequency. However, it is obvious that bias of sample collection can be diminished as the genotype frequency of the other genes studied in the same population was in HWE. The major limitation of the recent finding is the small study population size, especially in the male group. However, in the study of gender-dependent disease like OA this is quite expected.

\section{CONCLUSIONS}

In conclusion, we found that the nsSNP rs4747096 in ADAMTS14 was associated with female OA patients requiring total knee replacement in a Thai population. Therefore, the role of ADAMTS14 in OA seems to be remarkably gender-dependent. Additional investigations conducted with a larger sample size or different ethnicities are required to validate the present findings.

\section{ACKNOWLEDGMENTS}

Research supported by the Chulalongkorn University Graduate Scholarship to Commemorate the 72nd Anniversary of His Majesty King Bhumibol Adulyadej, the 90th Anniversary of Chulalongkorn University Fund (Ratchadaphiseksomphot Endowment Fund), the 
Thailand Research Fund, Integrated Innovation Academic Center: IIAC Chulalongkorn University Centenary Academic Development Project (\#CU56-HR05). the Higher Education Research Promotion and National Research University Project in Thailand, Office of the Higher Education Commission (\#HR1163A), and the Research Funds from the Faculty of Science, Chulalongkorn University.

\section{REFERENCES}

Apte SS (2004). A disintegrin-like and metalloprotease (reprolysin type) with thrombospondin type 1 motifs: the ADAMTS family. Int. J. Biochem. Cell Biol. 36: 981-985.

Attur M, Wang HY, Kraus VB, Bukowski JF, et al. (2010). Radiographic severity of knee osteoarthritis is conditional on interleukin 1 receptor antagonist gene variations. Ann. Rheum. Dis. 69: 856-861.

Cal S, Obaya AJ, Llamazares M, Garabaya C, et al. (2002). Cloning, expression analysis, and structural characterization of seven novel human ADAMTSs, a family of metalloproteinases with disintegrin and thrombospondin-1 domains. Gene 283: 49-62.

Colige A, Vandenberghe I, Thiry M, Lambert CA, et al. (2002). Cloning and characterization of ADAMTS-14, a novel ADAMTS displaying high homology with ADAMTS-2 and ADAMTS-3. J. Biol. Chem. 277: 5756-5766.

Dieppe P and Kirwan J (1994). The localization of osteoarthritis. Br. J. Rheumatol. 33: 201-203.

Dieppe PA and Lohmander LS (2005). Pathogenesis and management of pain in osteoarthritis. Lancet 365: 965-973.

Fernandes RJ, Hirohata S, Engle JM, Colige A, et al. (2001). Procollagen II amino propeptide processing by ADAMTS-3. Insights on dermatosparaxis. J. Biol. Chem. 276: 31502-31509.

Futreal PA, Coin L, Marshall M, Down T, et al. (2004). A census of human cancer genes. Nat. Rev. Cancer 4: 177-183.

Hojima Y, McKenzie JA, van der Rest M and Prockop DJ (1989). Type I procollagen N-proteinase from chick embryo tendons. Purification of a new 500-kDa form of the enzyme and identification of the catalytically active polypeptides. J. Biol. Chem. 264: 11336-11345.

Hojima Y, Morgelin MM, Engel J, Boutillon MM, et al. (1994). Characterization of type I procollagen N-proteinase from fetal bovine tendon and skin. Purification of the 500-kilodalton form of the enzyme from bovine tendon. J. Biol. Chem. 269: 11381-11390.

Hurskainen TL, Hirohata S, Seldin MF and Apte SS (1999). ADAM-TS5, ADAM-TS6, and ADAM-TS7, novel members of a new family of zinc metalloproteases. General features and genomic distribution of the ADAM-TS family. J. Biol. Chem. 274: 25555-25563.

Ikegawa S (2007). New gene associations in osteoarthritis: what do they provide, and where are we going? Curr. Opin. Rheumatol. 19: 429-434.

Kellgren JH and Lawrence JS (1957). Radiological assessment of osteo-arthrosis. Ann. Rheum. Dis. 16: 494-502.

Kevorkian L, Young DA, Darrah C, Donell ST, et al. (2004). Expression profiling of metalloproteinases and their inhibitors in cartilage. Arthritis Rheum. 50: 131-141.

Kuno K, Kanada N, Nakashima E, Fujiki F, et al. (1997). Molecular cloning of a gene encoding a new type of metalloproteinase-disintegrin family protein with thrombospondin motifs as an inflammation associated gene. $J$. Biol. Chem. 272: 556-562.

Lapiere CM, Lenaers A and Kohn LD (1971). Procollagen peptidase: an enzyme excising the coordination peptides of procollagen. Proc. Natl. Acad. Sci. U. S. A. 68: 3054-3058.

Le Goff C, Somerville RP, Kesteloot F, Powell K, et al. (2006). Regulation of procollagen amino-propeptide processing during mouse embryogenesis by specialization of homologous ADAMTS proteases: insights on collagen biosynthesis and dermatosparaxis. Development 133: 1587-1596.

Murphy G and Nagase H (2008). Reappraising metalloproteinases in rheumatoid arthritis and osteoarthritis: destruction or repair? Nat. Clin. Pract. Rheumatol. 4: 128-135.

Rodriguez-Lopez J, Pombo-Suarez M, Loughlin J, Tsezou A, et al. (2009). Association of a nsSNP in ADAMTS14 to some osteoarthritis phenotypes. Osteoarthritis Cartilage. 17: 321-327.

Rodriguez S, Gaunt TR and Day IN (2009). Hardy-Weinberg equilibrium testing of biological ascertainment for Mendelian randomization studies. Am. J. Epidemiol. 169: 505-514.

Sezgin M, Erdal ME, Altintas ZM, Ankarali HC, et al. (2007). Lack of association polymorphisms of the IL1RN, IL1A, and IL1B genes with knee osteoarthritis in Turkish patients. Clin. Invest. Med. 30: E86-E92.

Sherry ST, Ward MH, Kholodov M, Baker J, et al. (2001). dbSNP: the NCBI database of genetic variation. Nucleic Acids Res. 29: 308-311. 
Somerville RP, Longpre JM, Jungers KA, Engle JM, et al. (2003). Characterization of ADAMTS-9 and ADAMTS-20 as a distinct ADAMTS subfamily related to Caenorhabditis elegans GON-1. J. Biol. Chem. 278: 9503-9513.

Spector TD and Macgregor AJ (2004). Risk factors for osteoarthritis: genetics. Osteoarthritis Cartilage. 12 Suppl A: S39-S44.

Tammachote R (2011). The genetics behind osteoarthritis: Asian focus. Asian Biomed. 5: 23-36.

Teichtahl AJ, Wluka AE, Proietto J and Cicuttini FM (2005). Obesity and the female sex, risk factors for knee osteoarthritis that may be attributable to systemic or local leptin biosynthesis and its cellular effects. Med. Hypotheses 65: 312-315.

Tuderman L, Kivirikko KI and Prockop DJ (1978). Partial purification and characterization of a neutral protease which cleaves the N-terminal propeptides from procollagen. Biochemistry 17: 2948-2954.

Valdes AM and Spector TD (2009). The contribution of genes to osteoarthritis. Rheum. Dis. Clin. North Am. 34: 581-603. 